\title{
Summarizing bodies of evidence on the implementation and effectiveness of FP/RH interventions
}

Ian Askew

Population Council

Follow this and additional works at: https://knowledgecommons.popcouncil.org/departments_sbsr-rh

Part of the International Public Health Commons, Maternal and Child Health Commons, Quantitative, Qualitative, Comparative, and Historical Methodologies Commons, and the Women's Health Commons How does access to this work benefit you? Let us know!

\section{Recommended Citation}

Askew, lan. 2014. "Summarizing bodies of evidence on the implementation and effectiveness of FP/RH interventions," presentation at the Third Global Symposium on Health Systems Research, Cape Town, South Africa. 


\section{Summarizing bodies of evidence on the implementation and effectiveness of FP/RH}

interventions

\section{Ian Askew}

Improving recommendations for policies and practices to strengthen people-centered health systems:

Is the State of Evidence sufficient?

Satellite Session

Third Global Symposium on Health Systems Research

September 29, 2014, Cape Town, South Africa 


\section{Approaches to compiling and summarizing bodies of evidence}

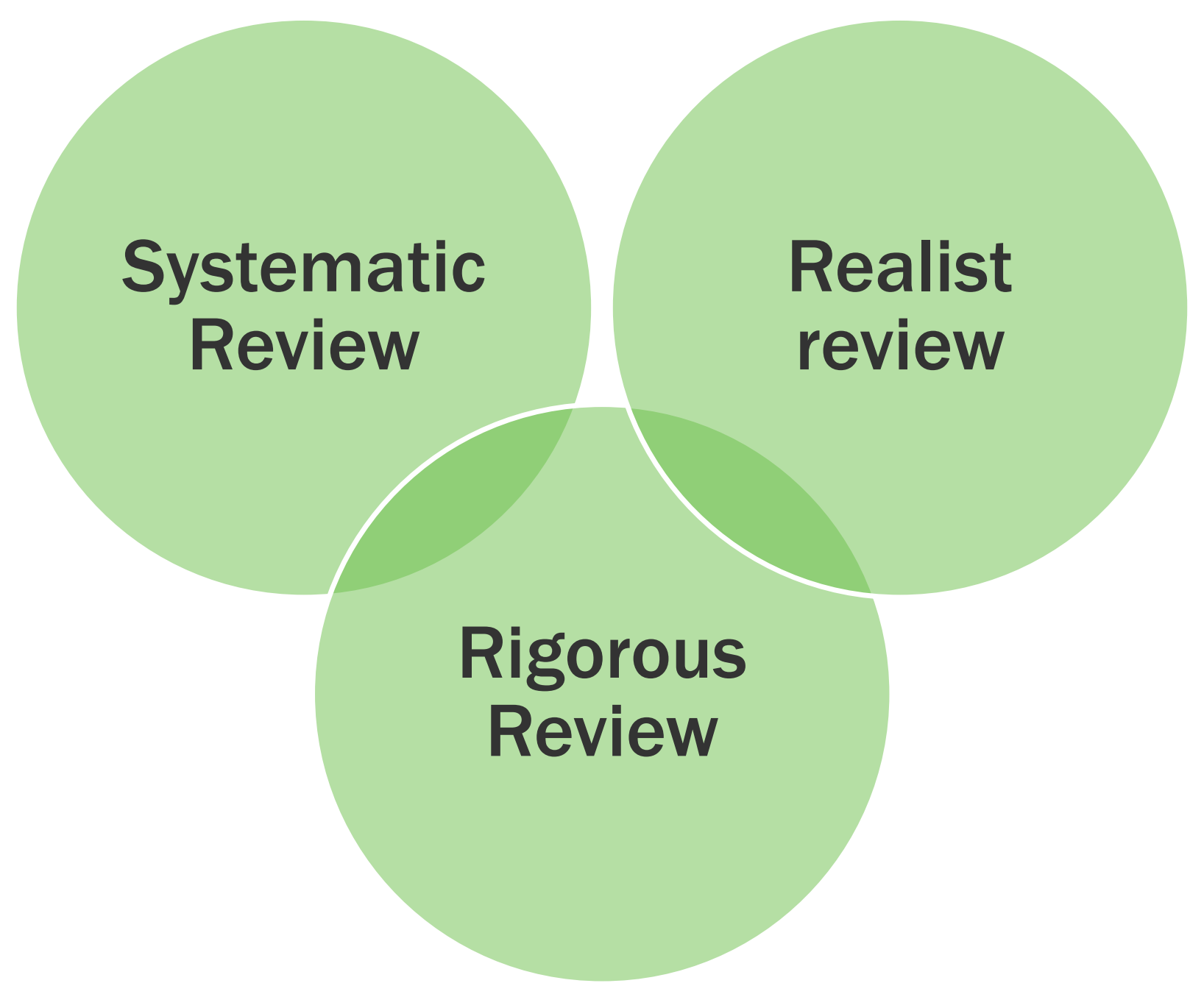




\section{Systematic Reviews}

"A systematic review is a scientific

investigation that focuses on a specific question and uses explicit, pre-specified scientific methods to identify, select, assess, and summarize the findings of similar but separate studies. It may include a quantitative synthesis (meta-analysis), depending on the available data" (Institute of Medicine, US National Academy of Sciences)
A systematic review can be defined as a explicit and systematic methods to identify, appraise and summarize the criteria. If this description predetermined methods) is not presiption (of the to make a thorougresent, it is not possible quality of the review" (UK National Institute fc

Clinical Excellence)

The key characteristics of a systematic review are:

- A clearly stated set of objectives with pre-defined eligibility criteria for including studies;

- An explicit, reproducible methodology;

- A systematic search that attempts to identify all studies that would meet the eligibility criteria;

- An assessment of the validity of the findings of the included studies;

- A systematic presentation, and synthesis, of the characteristics and findings of the included studies.

(Cochrane Review) 


\section{Systematic review process}

Step 1: Initiate the process:

-Step 2: Develop the review protocol:

-Step 3: Systematically locate, screen, and select the studies for review

-Step 4: Appraise the risk of bias in the individual studies and extract the data for analysis

-Step 5: Synthesize the findings and assess the overall quality of the body of evidence

-Step 6: Prepare a final report and have the report undergo peer review

Institute of Medicine 2011. Finding what works in health care: standards for systematic reviews, National Academy of Sciences 


\section{Systematic vs. Rigorous reviews}

\section{Systematic review}

Starts with a clear question/hypothesis

Team of authors including methodologists

Thorough literature search methods

Explicit inclusion and exclusion criteria

Assessment of risk of bias

Appraisal of strength of evidence e.g. GRADE

Managed conflicts of interest

\section{Rigorous / expert review}

May start with a general discussion

Authors are usually content experts

Does not always include literature search

Vague inclusion $+/$ - exclusion criteria

Bias not usually assessed

Limited formal appraisal of evidence

Conflicts of interest not always stated

Source: Isba 2013 


\section{Rigorous reviews using an evidence framework}

"A global health evidence framework [is] one which uses multiple domains to arrive at a summary judgment of the evidence for community or population health interventions or programs"

Source: Luoto et al, 2013
- Systematic and rigorous

- Transparent procedures

- Summary judgment

- Rating across multiple domains

- Quality, quantity, relevance, consistency, context....

- Focus on evidence of effectiveness of an intervention 


\section{Examples of domains for grading strength of evidence}

\section{USCPSTF}

\section{Execution}

Design suitability

Number of studies

Consistency

Effect size

Expert opinion

\section{DFID}

Number of studies

Quality of body of evidence

Context

Consistency

Diversity of methods
But....Evidence frameworks differ in terms of how domains are rated:

Classifying strength of evidence Magnitude of benefits vs. harms Consideration of context Implementation procedures Feasibility

- Costs

- Sustainability 


\section{Realist reviews}

- Identifies underlying causal mechanisms of a complex intervention and explores how they work within a specific context to produce particular outcome(s)

\section{Context + Mechanism = Outcomes}

- C-M-O configuration explains why and how an intervention works: Theory of Change ("program theory") 


\section{Example of a C-M-O Theory of Change}

\section{"In this context, that mechanism generates this outcome"}

For a fee-removal intervention:

"Poor couples who value family planning (C) are enabled $(M)$ to use contraception to space their pregnancies $(0)$ " 


\section{Approach to a realist review}

\begin{tabular}{l|l|}
\hline \multicolumn{1}{|c|}{ Stage } & \multicolumn{1}{c|}{ Action } \\
\hline Define the scope of the review & Identify the question \\
& Clarify the purpose of the review \\
& Find and articulate the ToCs \\
\hline $\begin{array}{l}\text { Search for and appraise the } \\
\text { evidence }\end{array}$ & Search for the evidence \\
\hline Extract and synthesize findings & Test of relevance \\
\hline
\end{tabular}

Develop narrative

Source: Rycroft-Malone et al., 2012 


\section{Which type of review and body of evidence for which type of recommendation?}

Efficacy of an intervention in meeting health needs of the individual / couple

- Service delivery guidelines

Effectiveness of delivering interventions at the population level

- Delivery programming guidance

Sustainability at national / programme level

- Systems strengthening and scale-up / mainstreaming

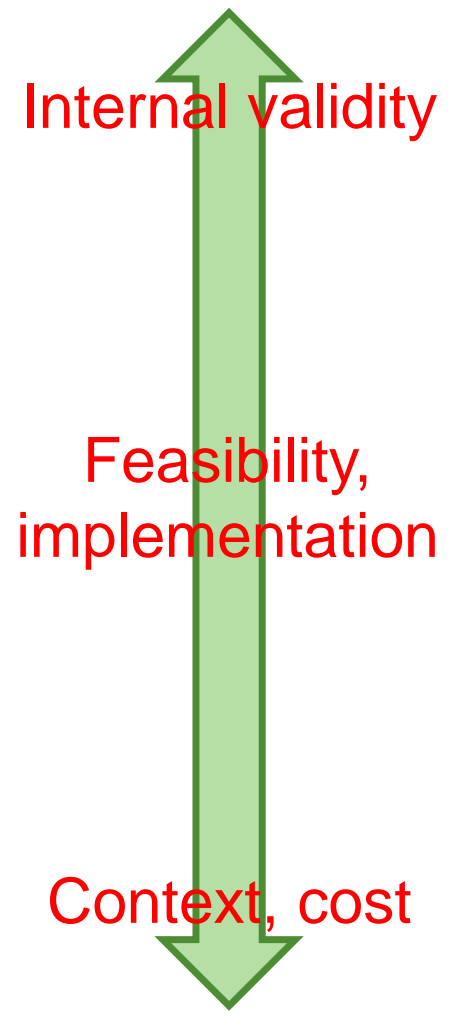




\section{Recommendations}

\section{STEP $\mathbb{S}_{\mathrm{g} U P}$}

SECOND CONSULTATION ON DEVELOPING STANDARDS FOR IDENTIFYING EVIDENCE-BASED PRACTICES IN REPRODUCTIVE HEALTH

18TH-19TH SEPTEMBER 2013

SELSDON PARK HOTEL, CROYDON, UK

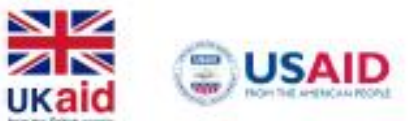




\section{Recommendations}

Bodies of evidence that inform decision-makers on the effectiveness of interventions are best summarized using a transparent, structured

review process that includes evidence from

both randomized and rigorous non-randomized designs with systematic comparisons

Bodies of evidence to inform implementation and scaling-up decisions can be derived from implementation research and economic evaluations. Highest-quality data are generated when the decision question is clearly stated and the research design tailored to generate evidence that will address that question

Such bodies of evidence should be guided by a theory of change, reviewed rigorously, synthesised systematically, and summarised to inform implementation decisions identified by decision-makers 


\section{Recommendations}

A systematic, transparent, and replicable process, guided by an explicit evidence framework, should be followed when developing practice recommendations from a body of evidence. The evidence framework should incorporate those domains that are of specific interest to particular decisionmakers; different evidence frameworks may be appropriate for summarising evidence to inform different types of decisions
Recommendation formulation should be carefully planned and implemented, using a representative and knowledgeable expert group and recommendation statements or diagrams that accurately and unequivocally represent the body of evidence available

Given the diversity of contexts in which RH/FP interventions are implemented, recommendations for implementation should offer a choice of options - that is, should be 'evidence-informed' - rather than specify a single 'evidence-based' recommendation for addressing a particular need or problem 